\title{
Title: S-acylation is a positive regulator of FLS2-mediated plant immunity
}

\author{
Authors: \\ Charlotte H. Hurst ${ }^{1,2}$, Dionne Turnbull ${ }^{1+}$, Julien Gronnier ${ }^{3,4^{+}}$, Sally Myles ${ }^{1}$, Robin L. Pflughaupt ${ }^{5}$, \\ Michaela Kopischke $^{6}$, Paul Davies ${ }^{5}$, Susan Jones7, Silke Robatzek ${ }^{6,8}$, Cyril Zipfel ${ }^{3,6}$, Piers A. Hemsley ${ }^{1,2^{*}}$
}

Affiliations:

${ }^{1}$ Division of Plant Sciences, School of Life Sciences, University of Dundee, Dow Street, Dundee, DD1 $5 \mathrm{EH}$, Scotland, UK.

${ }^{2}$ Cell and Molecular Sciences, The James Hutton Institute, Invergowrie, Dundee, DD2 5DA, Scotland, UK.

${ }^{3}$ Institute of Plant and Microbial Biology, Zurich-Basel Plant Science Center, University of Zurich, 8008 Zurich, Switzerland.

${ }^{4}$ Present address - ZMBP Universität Tübingen, Auf der Morgenstelle 32, D-72076 Tübingen, Germany

${ }^{5}$ Medical Research Council Protein Phosphorylation and Ubiquitylation Unit, School of Life Sciences, University of Dundee, Dow Street, Dundee, DD1 5EH, Scotland, UK.

${ }^{6}$ The Sainsbury Laboratory, University of East Anglia, Norwich Research Park, NR4 7UH, Norwich, England, UK.

7 Information and Computational Sciences, The James Hutton Institute, Invergowrie, Dundee, DD2 5DA, Scotland, UK.

${ }^{8}$ LMU Munich Biocenter, Großhadener Strasse 4, 82152 Planegg, DE.

$\dagger$ These authors contributed equally

* Author for correspondence - p.a.hemsley@dundee.ac.uk

Abstract: Plant receptor kinases are key transducers of extracellular stimuli and are regulated by numerous post-translational modifications. S-acylation involves the addition of long-chain fatty acids to cysteine residues within proteins, altering their biophysical properties. Here we identify S-acylation at a conserved cysteine of the receptor kinase FLS2 as crucial for function during plant immunity. We observe rapid S-acylation of FLS2 upon perception of its flg22 ligand in a BAK1 co-receptor dependent manner. Notably, S-acylation is essential for several aspects of FLS2-mediated early and late signalling, including anti-bacterial immunity. Biochemical analysis suggests that FLS2 S-acylation assists the stabilisation of activated receptor kinase protein complexes at the plasma membrane to increase signalling efficiency. 
Main text: The plasma membrane defines the boundary between the cell interior and the external environment, and is the perception site for most stimuli that a plant encounters. The receptor kinase (RK) FLAGELLIN SENSING 2 (FLS2) is the receptor for bacterial flagellin-derived flg22 (1). We recently discovered that FLS2, alongside all other plant RKs tested, is modified by S-acylation (2). S-acylation is a fatty acid-based post-translational modification (3) that can change the physical properties of a protein. This modification can lead to changes in protein trafficking, stability, and turnover, and has been hypothesised to be a driver of membrane partitioning (4). Changes in protein S-acylation state have also been proposed to modulate protein-protein and protein-membrane interactions, and even alter protein activation states (5).

Our previous analysis of FLS2 identified the juxta-transmembrane (TM) domain cysteines (Cys830,831) as being constitutively S-acylated, but this modification was dispensable for FLS2 function (2). All RK superfamily members tested, with or without a juxta-TM S-acylation site homologous to FLS2 C $C^{830,831}$, also appear to be S-acylated (2). This indicates that non-juxta-TM S-acylation sites, potentially conserved in all RKs, exist. Notably, FLS2 S-acylation increased by almost $60 \%$ in Col-0 wild-type plants following 20-min exposure to flg22 compared to mock treatment (figures $1 \mathrm{~A}$ and $\mathrm{S} 1 \mathrm{~A}$ ) and returned to basal levels within $1 \mathrm{~h}$. Treatment of Arabidopsis Col-0 plants with elf18, a conserved peptide derived from bacterial elongation factor Tu recognised by the RK EFR (ELONGATION FACTOR-Tu RECEPTOR) that acts similarly to FLS2 (6), failed to elevate FLS2 S-acylation (figure 1B) (7). This demonstrates that the increase in FLS2 S-acylation is ligand-dependent rather than a general phenomenon related to activation of RK-mediated defence responses.

FLS2 $\mathrm{C}^{830,831} \mathrm{~S}$ mutants (2) showed a similar elevation of S-acylation in response to flg22 (figure S1B). FLS2 therefore contains S-acylation sites in addition to $C^{830,831}$ that are responsive to ligand perception. Interestingly, 1-2 conserved cysteine residues at the C-terminus of the kinase domain (corresponding to FLS2 Cys 1132 and/or 1135, figure S2) are conserved across RKs in Arabidopsis thaliana and the broader Streptophyte lineage, suggesting a conserved and important role for these cysteines. FLS2 $\mathrm{C}^{830,831} \mathrm{~S}$ is weakly S-acylated in the absence of flg22 when overexpressed in Nicotiana benthamiana. Mutation of FLS2 Cys 1132 and 1135 in addition to Cys 830 and 831 (FLS2 C C30,831,1132,1135S) abolished FLS2 S-acylation compared to FLS2 $\mathrm{C}^{830,831} \mathrm{~S}$ (figure $1 \mathrm{C}$ ) when expressed in $\mathrm{N}$. benthamiana, suggesting that Cys 1132 and 1135 are sites of S-acylation. Accordingly, fls2c/proFLS2:FLS2 C1132,1135S Arabidopsis plants (figure S3) showed no increase in flg22-induced S-acylation (figure 1D). Consistent with its ligand-dependency, FLS2 S-acylation was dependent on its co-receptor BAK1 (BRI1-ASSOCIATED KINASE) (figure 1E). Additionally, FLS2 flg22 induced S-acylation was unaffected in chc2-1 mutants (8) of clathrin heavy chain 2, indicating that S-acylation occurs before endocytosis (figure 1E). A recent proteomic analysis of S-acylated proteins from Arabidopsis also identified these C-terminal cysteines as putative S-acylation sites in a number of RKs unrelated to FLS2 (9). These data provide independent support to our hypothesis that these conserved cysteines are a site of S-acylation within the RK superfamily.

Consistent with the conservation of the S-acylated cysteines among RKs, fls2c/proFLS2:FLS2 $C^{1132,1135 S}$ plants are impaired in several aspects of early immune signalling, such as reactive oxygen species production, MAP kinase activation and immune gene expression (figure 2). Similarly, later flg22induced immune outputs, such as PR1 (PATHOGENESIS-RELATED GENE 1) expression and seedling growth inhibition were affected in fls2c/proFLS2:FLS2 $C^{1132,1135} S$ plants (figure 3A, B). Consistent with 
these signalling defects, FLS2 $\mathrm{C}^{1132,1135} \mathrm{~S}$ failed to complement the hyper-susceptibility of fls2 mutant plants to the pathogenic bacterium Pseudomonas syringae pv. tomato (Pto) DC3000 (figure 3C). Structural homology modelling indicates that the $C^{1132,1135} S$ mutation is unlikely to affect FLS2 protein integrity, structure or kinase activity (figure S4). Together, these data show that FLS2 S-acylation at Cys $^{1132,1135}$ is necessary for FLS2 function during plant immunity.

Assessment of flg22-induced FLS2-BAK1 complex formation by co-immunoprecipitation following solubilisation with either cold non-ionic IGEPAL CA-630 detergent or the nanodisc-forming diisobutylene/maleic acid (DIBMA) copolymer (10) indicated that BAK1 recruitment was reduced in FLS2 $C^{1132,1135}$ S mutants (figure 4A, B). Furthermore, flg22-induced BAK1 $\mathrm{S}^{612}$ auto-phosphorylation (11) was consistently weaker in FLS2 $\mathrm{C}^{1132,1135}$ S-expressing plants (figure $4 A$ ). The apparent reduction in FLS2 $\mathrm{C}^{1132,1135}$ S-BAK1 interaction was not due to altered abundance in unstimulated Arabidopsis plants, as FLS2 and FLS2 $\mathrm{C}^{1132,1135} \mathrm{~S}$ accumulates to similar levels (figure S3). Both FLS2 and FLS2 $\mathrm{C}^{1132,1135} \mathrm{~S}$ show similar accumulation at the plasma membrane, lateral membrane mobility (figure S5), and association with REM1.3 nanodomains (figure S6), indicating that there is no aberrant basal cellular behaviour of the FLS2 $\mathrm{C}^{1132,1135} \mathrm{~S}$ mutant. FLS2 $\mathrm{C}^{1132,1135} \mathrm{~S}$ LRRs (leucine rich repeats) should bind flg22 and the BAK1 LRRs with similar efficiency to FLS2. The reduced FLS2 $C^{1132,1135}$ S-BAK1 complex formation observed therefore suggests that flg22-induced association between FLS2 and BAK1 is stabilised by additional S-acylation-dependent factors. The RKs PSKR1 (PHYTOSULFOKINE SIGNALLING PEPTIDE RECEPTOR KINASE) and SERK1 (SOMATIC EMBRYOGENESIS RECEPTOR KINASE 1) - the receptor and co-receptor for the plant peptide phytosulfokine ligand - have an in vitro Kd of $\sim 1 \mu \mathrm{M}$ and in vivo $\mathrm{Kd}$ of $\sim 4 \mathrm{nM}(12)$ in the presence of phytosulfokine, suggesting ternary RK LRR-ligand complexes are further stabilised by membrane composition (13), sterol content (14) or accessory proteins (15). S-acylation can affect protein partitioning in cold, non-ionic detergent-resistant membrane preparations (16). Following flg22 treatment, FLS2 $\mathrm{C}^{1132,1135} \mathrm{~S}$ occupancy in cold IGEPAL CA630 derived detergent resistant membranes (DRM) decreased (figure 4C, D). IGEPAL CA-630 mediated FLS2-BAK1 co-IP and DRMs data (figure 4A, C, D) suggests that the observed FLS2-BAK1 interaction is stabilised by an S-acylation-dependent molecular "shell". DIBMA copolymer does not produce a biphasic DRM/DSM-like system during solubilisation. Instead, DIBMA disrupts lipid-lipid, but not lipidprotein or protein-protein interactions toform native membrane nanodiscs (10). The reduced FLS2 $\mathrm{C}^{1132,1135} \mathrm{~S}-\mathrm{BAK} 1$ interaction in DIBMA (figure 4B) suggests that FLS2 S-acylation promotes closer protein packing of the activated complex. Altogether, our data suggest that flg22-induced, BAK1dependent S-acylation of FLS2 acts as a molecular checkpoint, providing stabilization for signallingactive molecular assemblies.

Recently, a pair of Asp-His-His-Cys motif containing (DHHC) S-acylating enzymes, PAT9 and PAT5, were identified as substrates of P2K1/DORN1/LecRK-I.9 RK kinase activity and were proposed to S-acylate P2K1 in response to extracellular ATP during innate immune signalling (17). Interestingly, and in contrast to our data with FLS2, S-acylation of P2K1 was proposed to inhibit immune signalling. The Sacylation sites proposed for $\mathrm{P} 2 \mathrm{~K} 1$ are at the $\mathrm{N}$-terminus of the kinase domain (17), do not appear to be conserved outside of the LecRK family and are distinct from the site identified here that is found in all RKs, including P2K1. This suggests that, consistent with our previous findings on FLS2 (2), and in common with other post-translational modifications, it is possible for S-acylation to affect multiple sites within an RK and have differing effects on RK function. Given the conserved nature of the S- 
acylation site in FLS2 identified here amongst RKs, this opens up the exciting possibility that S-acylation at this site may positively regulate the function of all RKs in a similar manner to FLS2.

\section{CRediT statement}

Charlotte H. Hurst: Conceptualization, Methodology, Validation, Formal analysis (Equal), Investigation (Lead), Data curation (Equal), Writing - Review \& Editing, Visualization. Dionne Turnbull: Methodology, Validation, Investigation (Equal), Writing - Review \& Editing. Julien Gronnier: Methodology, Formal analysis (Equal), Investigation (Equal), Data curation (Equal), Writing - Review \& Editing, Visualization, Funding acquisition (Equal). Sally Myles: Validation, Investigation. Michaela Kopischke: Investigation. Susan Jones: Methodology, Software, Investigation. Silke Robatzek: Resources, Writing - Review \& Editing, Supervision, Funding acquisition (Equal). Cyril Zipfel: Resources, Writing - Review \& Editing, Supervision, Funding acquisition (Equal). Piers A. Hemsley: Conceptualization (Lead), Methodology (Lead), Validation, Formal analysis (Lead), Investigation, Data curation (Lead), Resources, Writing - Original Draft (Lead), Writing - Review \& Editing (Lead), Visualization (Lead), Supervision (Lead), Project administration (Lead), Funding acquisition (Equal).

\section{Acknowledgements:}

We would like to thank Antje Heese for critical discussions and advice during the preparation of this manuscript. This work was supported by BBSRC EASTBIO-DTP studentship (grant number $\mathrm{BB} / \mathrm{M010996/1)}$ to SM and PH, BBSRC grants BB/M024911/1 and BB/P007902/1 to PH, Royal Society Grant RG140531 to PH, a Heisenberg fellowship from the Deutsche Forschungsgemeinschaft to SR, the Gatsby Charitable Foundation, the University of Zürich, the European Research Council under the Grant Agreement 773153 (grant IMMUNO-PEPTALK) to CZ, and the European Molecular Biology Organization (EMBO Long-Term Fellowship 438-2018) to JG. S.J. was supported by the Scottish Government's Rural and Environment Science and Analytical Services division (RESAS).

\section{Supplementary materials:}

Fig. S1 to S6

Materials and methods 
A

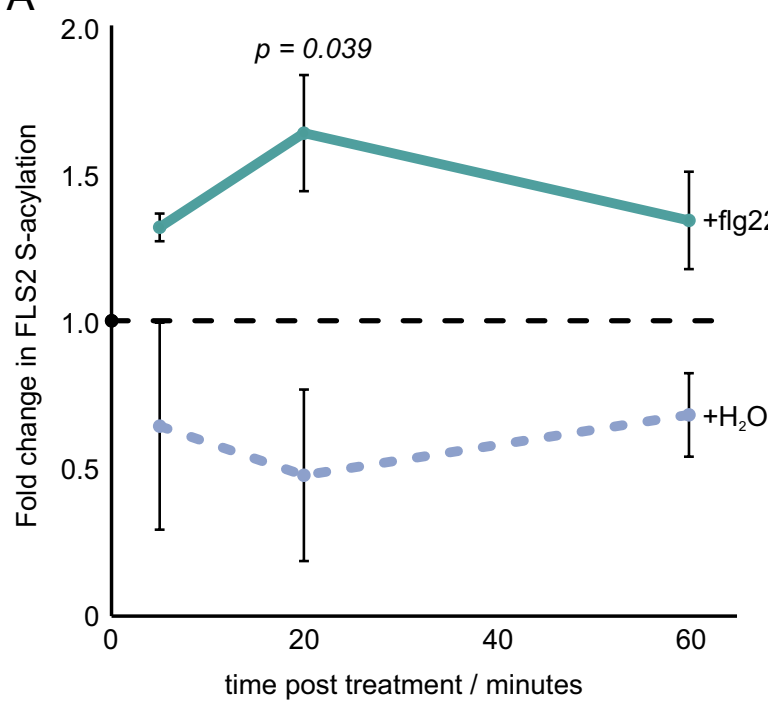

$\mathrm{D}$

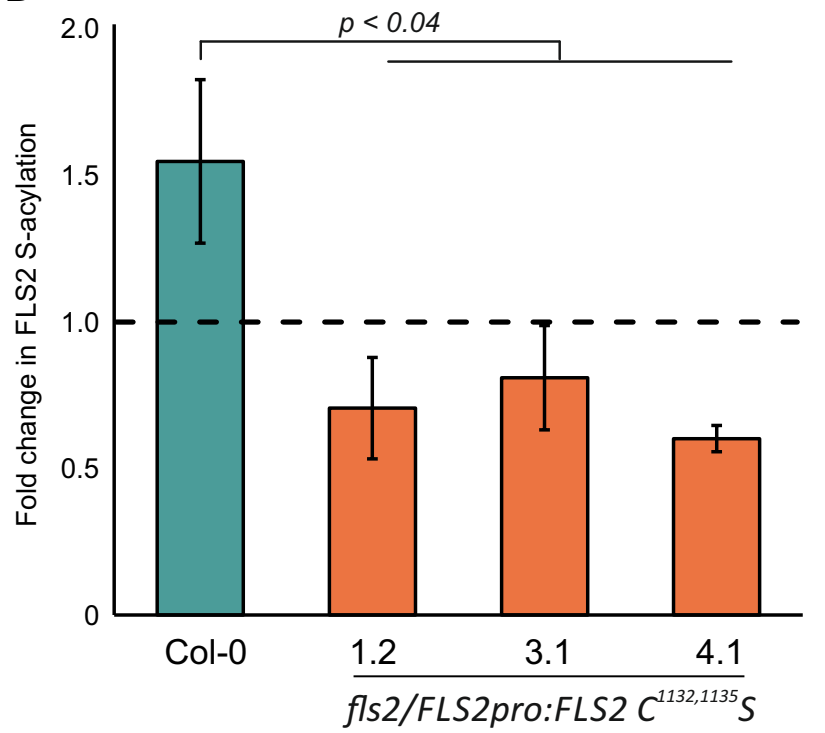

B

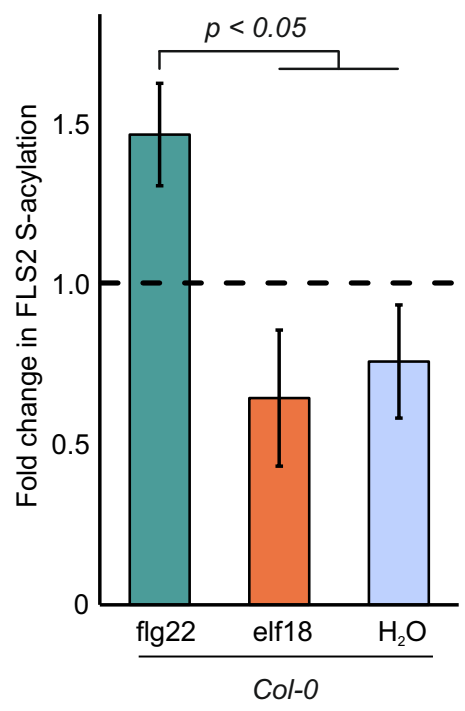

C

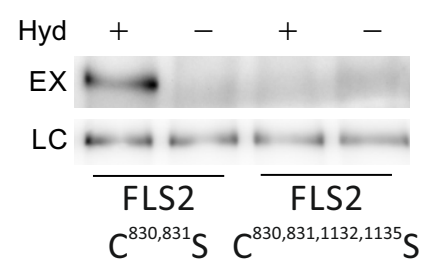

E

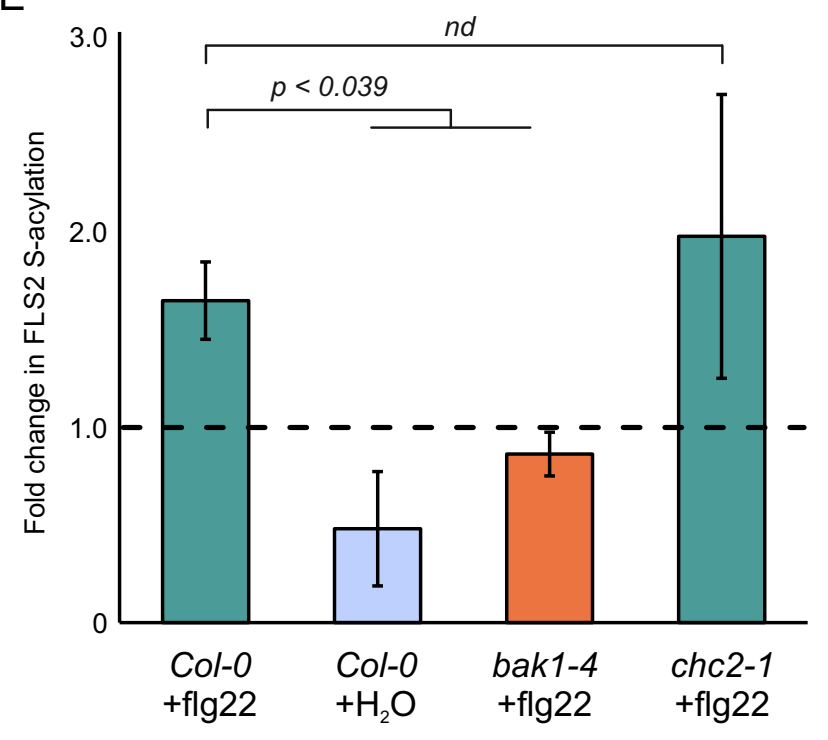

Figure 1. FLS2 S-acylation increases upon flg22 perception. A. Quantification of changes in FLS2 Sacylation following flg22 ( $n=5$, green solid line) or water only control $(n=2$, blue short dashed line) treatment. S-acylation state is shown relative to $\mathrm{T}_{0}$ (black, long dashed line). Error bars show SEM. Significance of difference between flg22 and water treated at 20 minutes is shown as determined by Student's t-test. B. FLS2 undergoes S-acylation in response to flg22 treatment but not elf18. S-acylation state is shown relative to untreated plants (black, dashed line). Error bars show SEM, $n=2$, significant differences to flg22 treated Col-O as determined by Student's t-test are shown. C. Mutation of FLS2 Cys1132,1135 to serine abolishes residual S-acylation observed in the FLS2 $C^{830,831} S$ background. EX indicates S-acylation state, LC - loading control, Hyd - indicates presence (+) or absence (-) of hydroxylamine. D. FLS2 $\mathrm{C}^{1132,1135} \mathrm{~S}$ mutants are blocked in flg22 mediated increases in S-acylation. Sacylation state is shown relative to water treated plants of the same genotype (black, dashed line). Error bars show SEM, $n=3$, significant difference to flg22 treated Col-0 as determined by Student's t-test are shown. E. S-acylation of FLS2 in response to flg22 requires BAK1. S-acylation state is shown relative to water treated plants of the same genotype (dashed line). Error bars show SEM, Col- $0+f l g 22 n=5, \mathrm{Col}-0+$ $\mathrm{H} 2 \mathrm{O} n=2$, bak1-4 $\mathrm{n}=4$, chc2-1 $\mathrm{n}=2$, significant difference to flg22 treated Col-O as determined by Student's t-test are shown. Data shown in panels $A$ and $E$ are derived from the same biological repeats, Col0 controls are therefore shared between panels. 
A

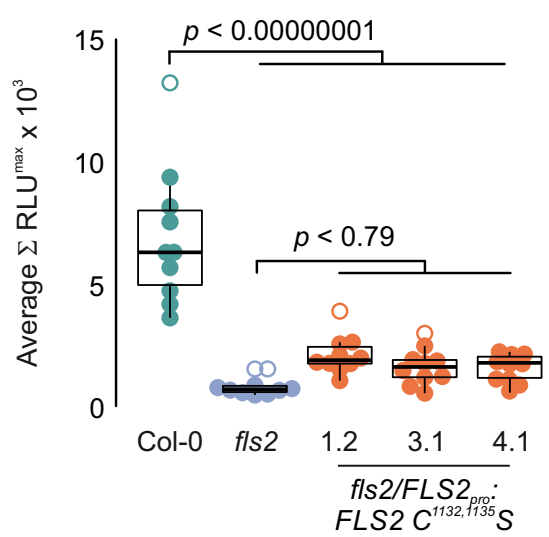

B

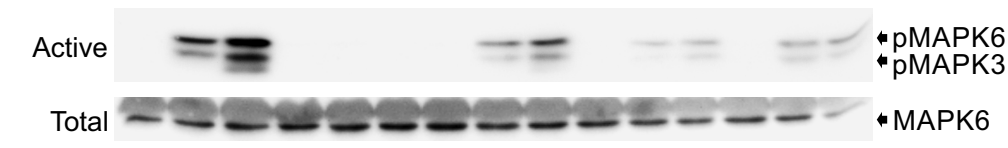

Minutes $\frac{0 \quad 5 \quad 15}{\text { Col-0 }} \frac{0 \quad 5 \quad 15}{f / s 2} \frac{0 \quad 5 \quad 15}{1.2} \frac{0 \quad 5 \quad 15}{3.1} \frac{0 \quad 5 \quad 15}{4.1}$

fls2/FLS2 $2_{\text {pro }}: F L S 2 C^{1132,1135} S$
C

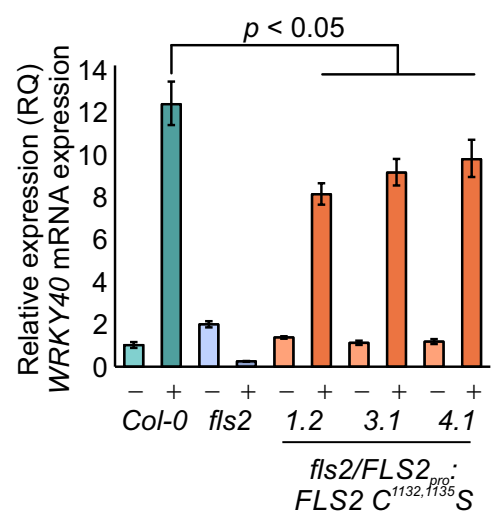

D

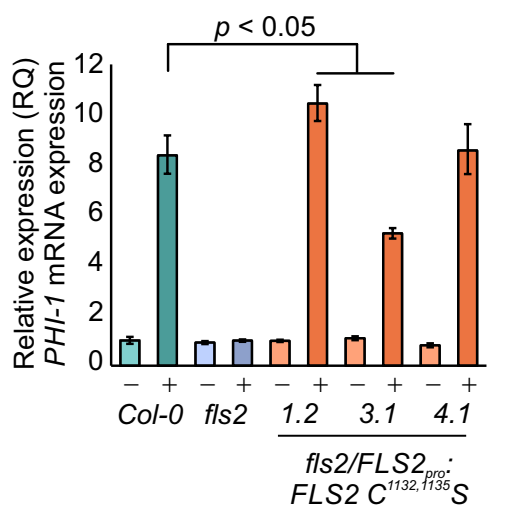

Figure 2. Acute responses to flg22 perception are reduced in FLS2 $C^{1132,1135}$ S expressing plants. A. ROS production induced by $100 \mathrm{nM}$ flg22 treatment. Data points are the sum of the 3 highest consecutive readings per sample. $n=10$ per genotype. Statistical outliers are shown as open circles. Box shows median and IQR, whiskers show +/- 1.5 x IQR. Statistical differences were calculated using ANOVA and Tukey HSD tests. B. MAPK activation in fls2/FLS2pro:FLS2 $C^{1132,1135} S$ seedlings in response to $100 \mathrm{nM}$ flg22 as determined over time by immunoblot analysis. pMAPK6/pMAPK3 show levels of active form of each MAPK. MAPK6 indicates total levels of MAPK6 as a loading control. Upper shadow band in MAPK6 blot is RUBISCO detected non-specifically by secondary antibody. C. Induction of WRKY40 gene expression after 1 hour treatment with $1 \mu \mathrm{M}$ flg22 in fls2/FLS2pro:FLS2 $C^{1132,1135} S$ seedlings as determined by qRTPCR. D. Induction of $P H I-1$ gene expression after 1 hour treatment with $1 \mu \mathrm{M}$ flg22 in fls2/FLS2pro:FLS2 $C^{1132,1135} S$ seedlings as determined by qRT-PCR. Values were calculated using the $\Delta \Delta C_{T}$ method, error bars represent $R Q_{\text {MIN }}$ and $R Q_{\text {MAX }}$ and constitute the acceptable error level for a $95 \%$ confidence interval according to Student's t-test. Significant differences in transcript mRNA detected in fls2/FLS2pro:FLS2 $C^{1132,1135} S$ seedlings compared to Col-0 levels in flg22 treated samples are indicated. 

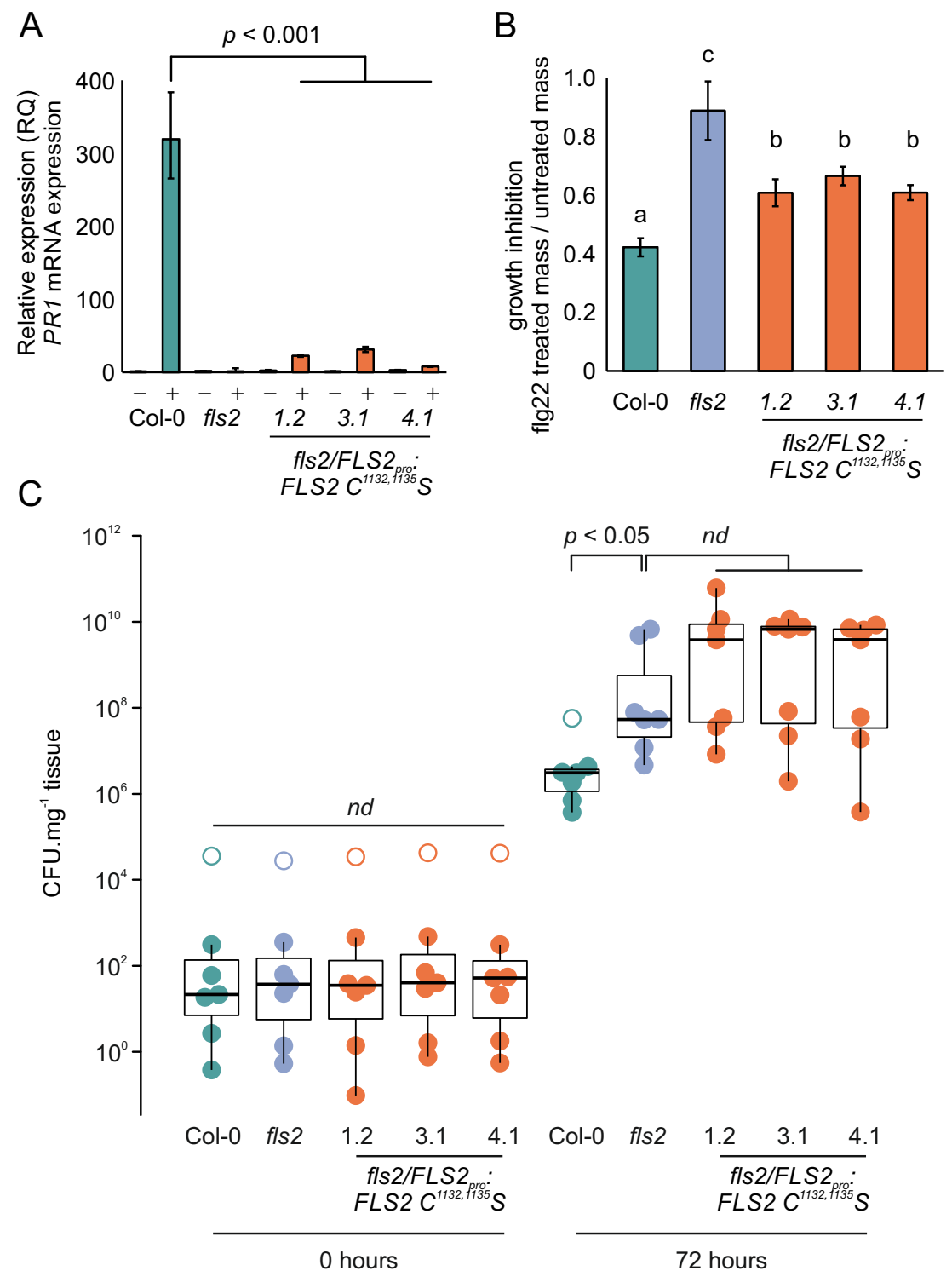

Figure 3. FLS2 S-acylation is required for long term immune response outputs (A). Induction of $P R 1$ gene expression after 24 hours treatment with $1 \mu \mathrm{M}$ flg22 in fls2/FLS2pro:FLS2 $C^{1132,1135} S$ seedlings as determined by qRT-PCR. Values were calculated using the $\triangle \Delta C_{T}$ method, error bars represent RQMIN and RQMAX and constitute the acceptable error level for a 95\% confidence interval according to Student's ttest. Significant differences in transcript mRNA detected in fls2/FLS2pro:FLS2 $C^{1132,1135} S$ seedlings compared to Col-0 levels in flg22 treated samples are indicated. Similar data were obtained over 3 biological repeats. (B). Inhibition of growth after 10 days of $1 \mu \mathrm{M}$ flg22 treatment is reduced in fls2/FLS2pro:FLS2 $C^{1132,1135} S$ seedlings. Data are averages of 3 biological replicates, error bars are SEM, significant differences at $p<0.01$ are indicated $(a, b, c)$ and calculated by ANOVA with Tukey HSD test. (C). Resistance to $P$. syringae DC3000 infection is impaired by loss of FLS2 S-acylation. Box and whisker plots show data from 7 biological repeats (box denotes median and IQR, whiskers show +/- $1.5 \times$ IQR, outliers are shown as open circles), significance of differences calculated using one-way ANOVA and Tukey HSD tests. 
bioRxiv preprint doi: https://doi.org/10.1101/2021.08.30.457756: this version posted August 31, 2021. The copyright holder for this preprint (which was not certified by peer review) is the author/funder, who has granted bioRxiv a license to display the preprint in perpetuity. It is A made available under aCC-BY-NC-ND 4.0 International license.

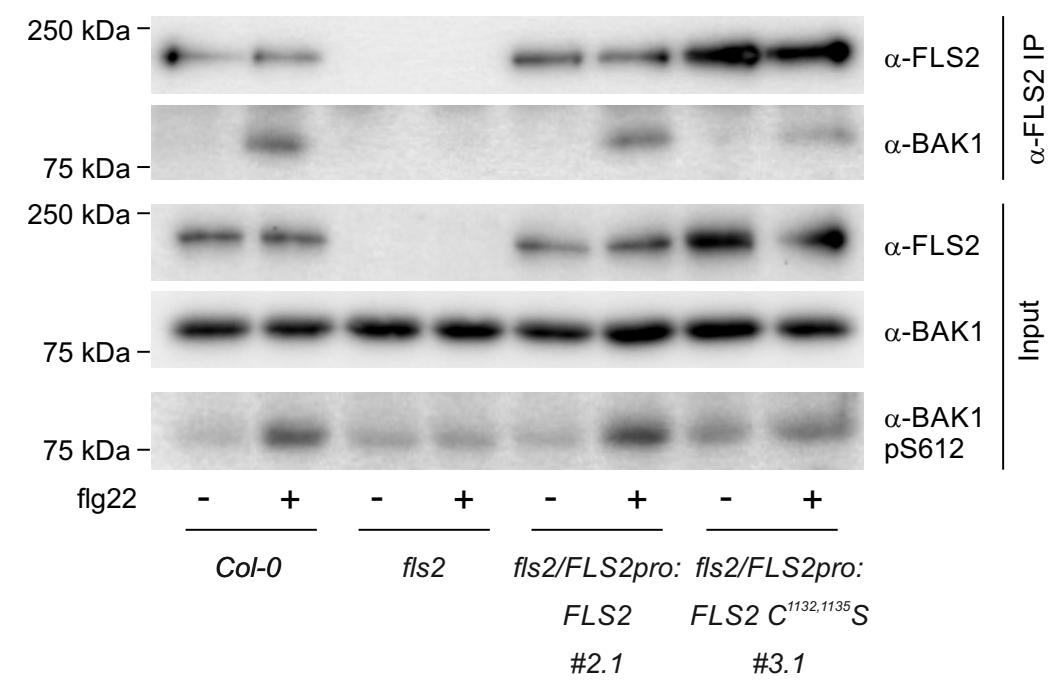

B

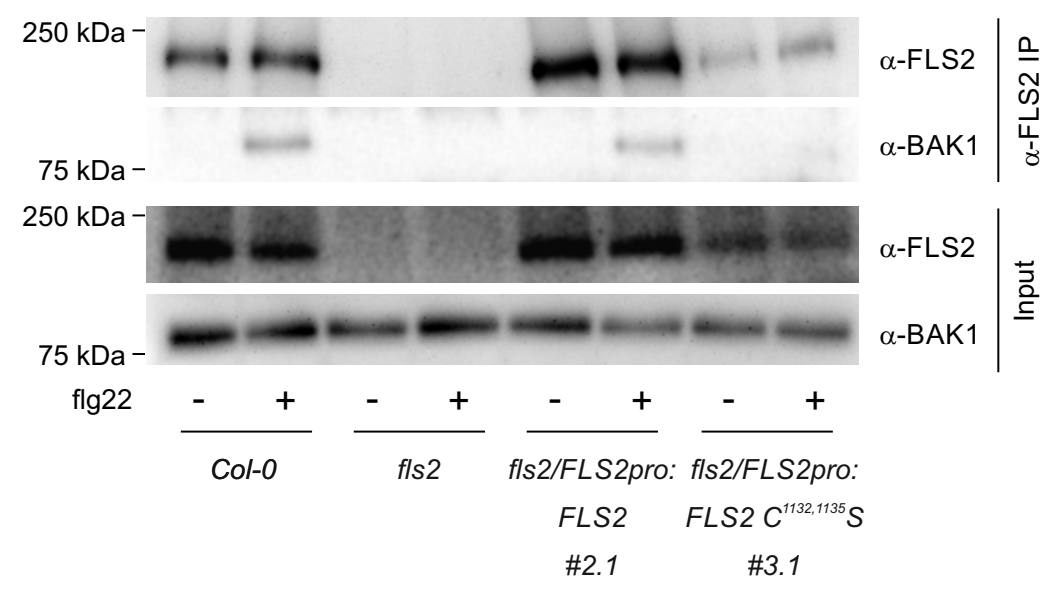

C

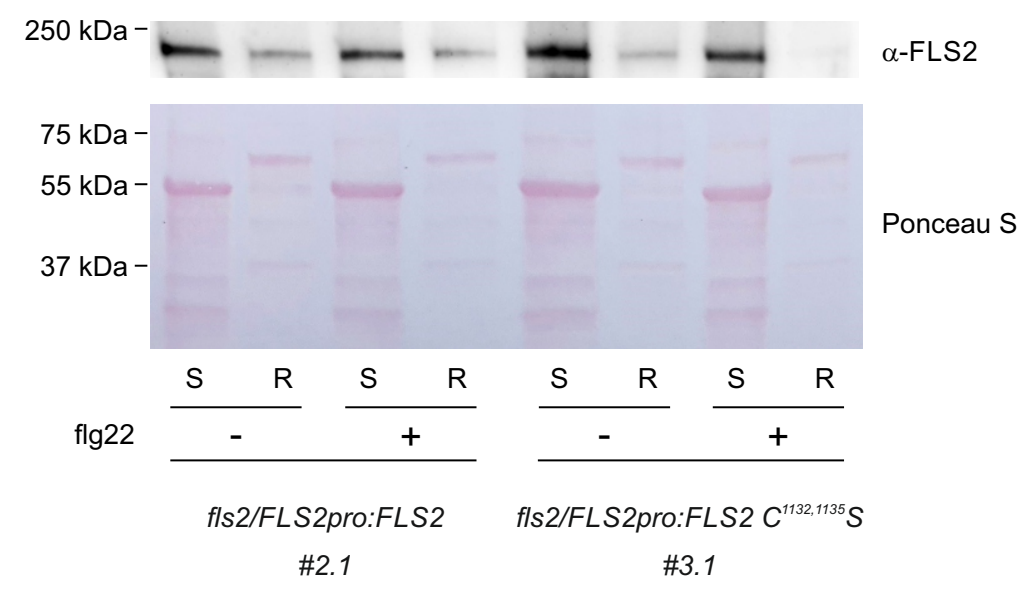

$\mathrm{D}$

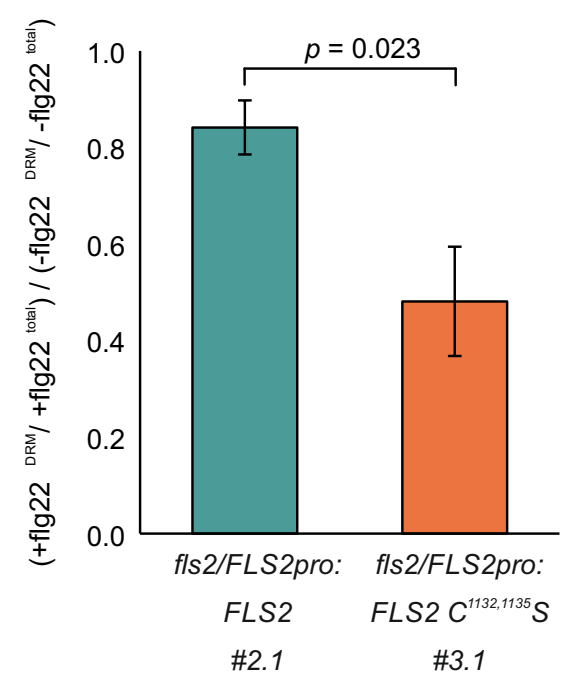

Figure 4. FLS2 $\mathrm{C}^{1132,1135} \mathrm{~S}$ shows reduced interaction with BAK1 following flg22 stimulation. A. FLS2 was immunoprecipitated from IGEPAL CA-630 solubilised flg22 treated Arabidopsis seedling lysates using anti-FLS2 rabbit polyclonal antibody. BAK1 recovery was assessed using rabbit polyclonal anti-BAK1 antibody. flg22 induced BAK1 autophosphorylation at Ser612 was assessed in input samples using rabbit polyclonal anti-BAK1 pS612 antibody. B. FLS2 was immunoprecipitated from DIBMA solubilised flg22 treated Arabidopsis seedling lysates using anti-FLS2 rabbit polyclonal antibody. BAK1 recovery was assessed using rabbit polyclonal anti-BAK1 antibody. C. Arabidopsis flg22 treated seedlings were lysed in cold IGEPAL CA-630 buffer and separated into detergent soluble (S) and detergent resistant (R) fractions. Relative partitioning of FLS2 into each fraction was determined by western blotting with anti-FLS2 rabbit polyclonal antibody. Loading and purity of fractions is shown by Ponceau S staining of the membrane. D. Quantification of data shown in C from 3 biological repeats. Error bars show SEM, significance was calculated using Student's t-test. 
bioRxiv preprint doi: https://doi org/10.1101/2021.08.30 457756. this version posted August 31, 2021. The copyright holder for this preprint (which was not certified by peer review) is the author/funder, who has granted bioRxiv a license to display the preprint in perpetuity. It is made available under aCC-BY-NC-ND 4.0 International license.

A

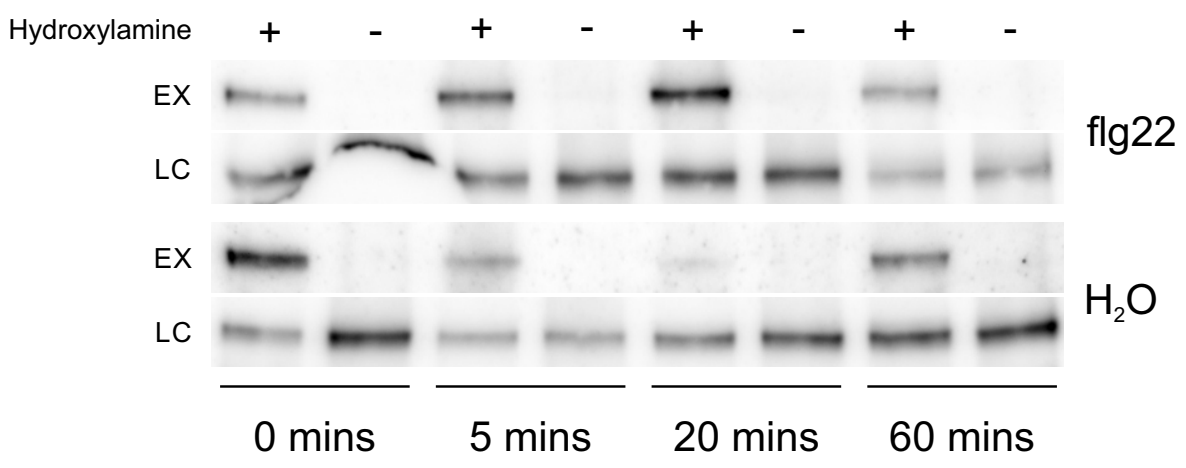

B

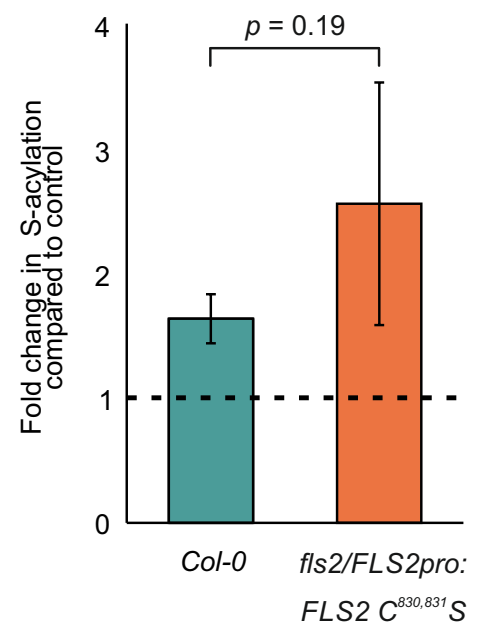

Supplemental figure 1. A. Example western blot from Col-0 plants treated with or without flg22 used to generate data shown in Figure 1. FLS2 S-acylation state is shown as a function of recovery on thiopropyl-Sepharose beads in the presence of hydroxylamine $(E X+)$. Samples lacking hydroxylamine (EX-) demonstrate completeness of blocking and lack of background or nonspecific binding. LC lanes act as input loading controls for standardisation. B. Quantification of changes in FLS2 and FLS2 $\mathrm{C}^{830,831} \mathrm{~S}$ S-acylation following flg22 treatment or water only control $(\mathrm{n}=$ 2 , blue short dashed line) treatment. flg22 induced changes in S-acylation state are shown relative to water only treatment (black dashed line). Col-O $n=5, F L S 2 C^{830,831} S n=2$, error bars show SEM. Significance of difference in S-acylation state change between FLS2 $(\mathrm{CO} /-0)$ and FLS2 $\mathrm{C}^{830,831} \mathrm{~S}$ is shown as determined by Student's t-test. 

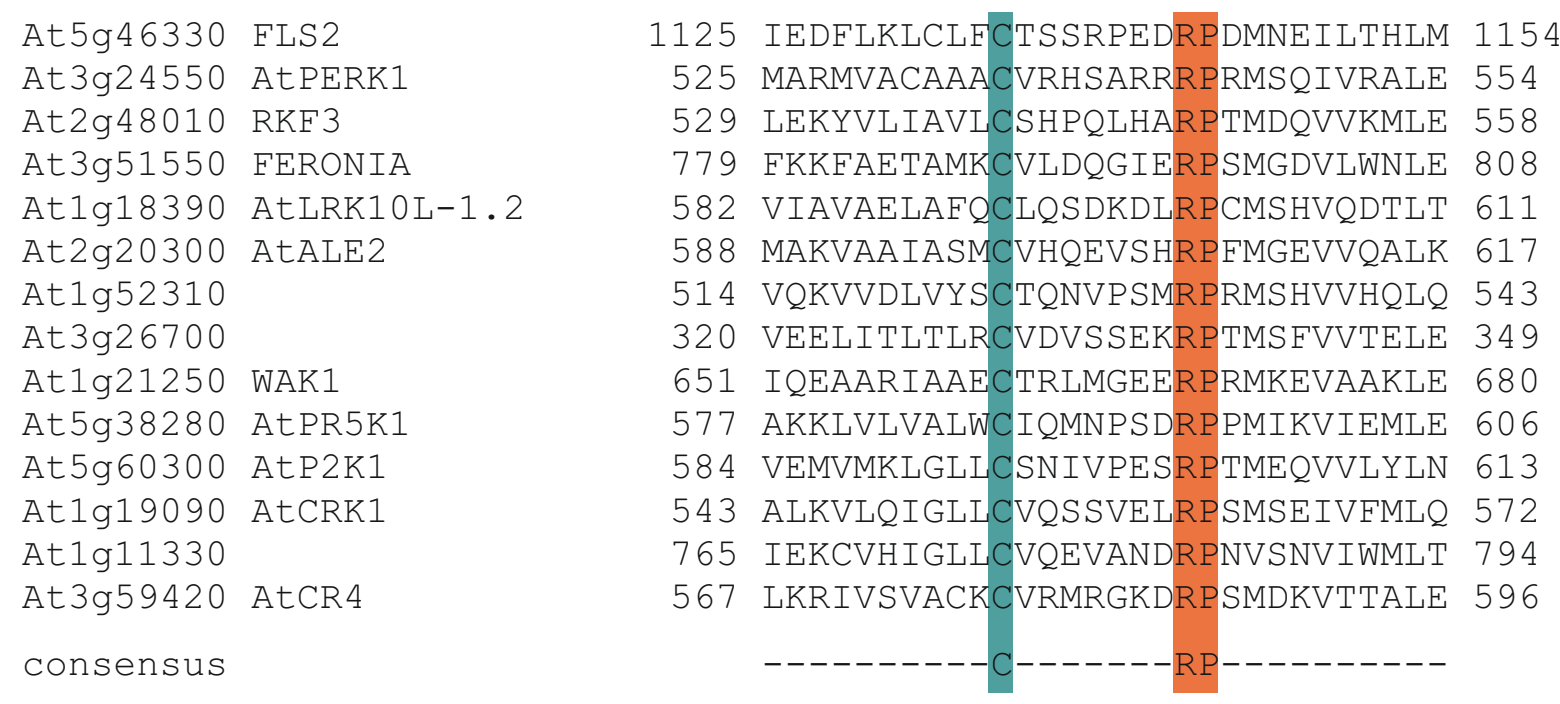

Supplemental figure 2. Alignment of representative members of the wider Arabidopsis RK superfamily, centred on the conserved $\mathrm{C}[\mathrm{X}]_{7} \mathrm{RP}$ motif found in the loop between the $\mathrm{G}-$ and $\mathrm{H}$ helices of the kinase domain, and identified as containing at least one S-acylated cysteine. 


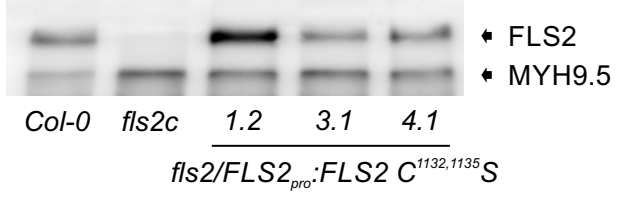

Supplemental figure 3. Expression levels of FLS2 $\mathrm{C}^{1132,1135} \mathrm{~S}$ in transgenic lines used in this study. $50 \mu \mathrm{g}$ total protein from 7 day old seedlings was loaded per lane. MYH9.5 is a previously reported cross-reacting protein with the primary anti-FLS2 antibody used . 
A

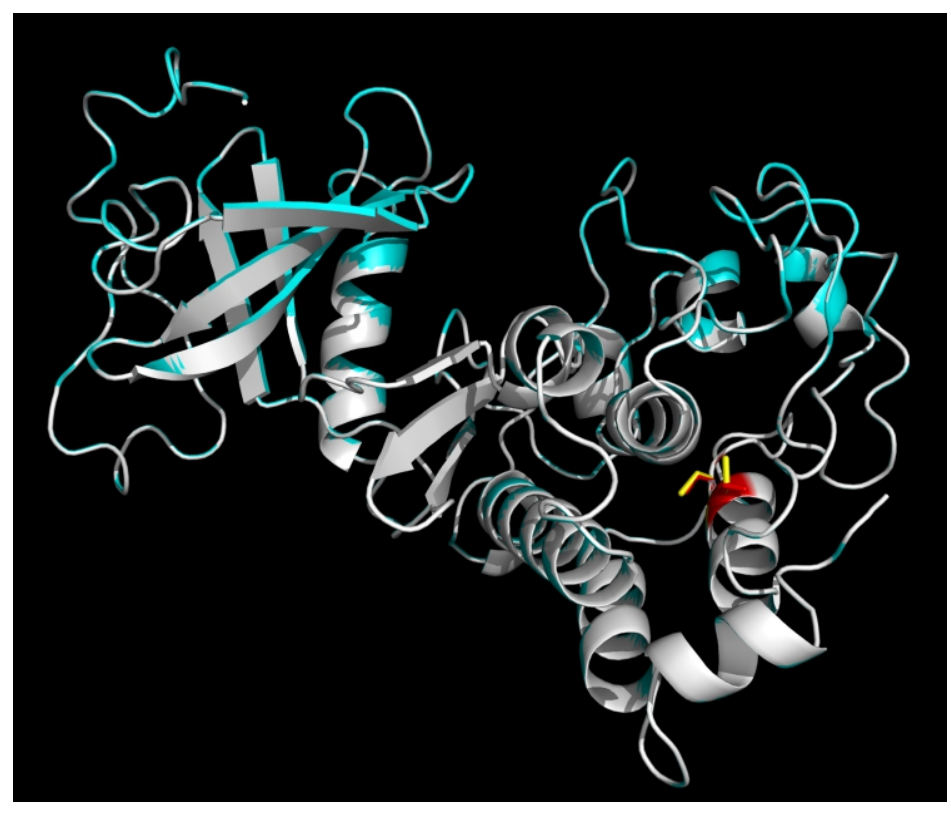

B

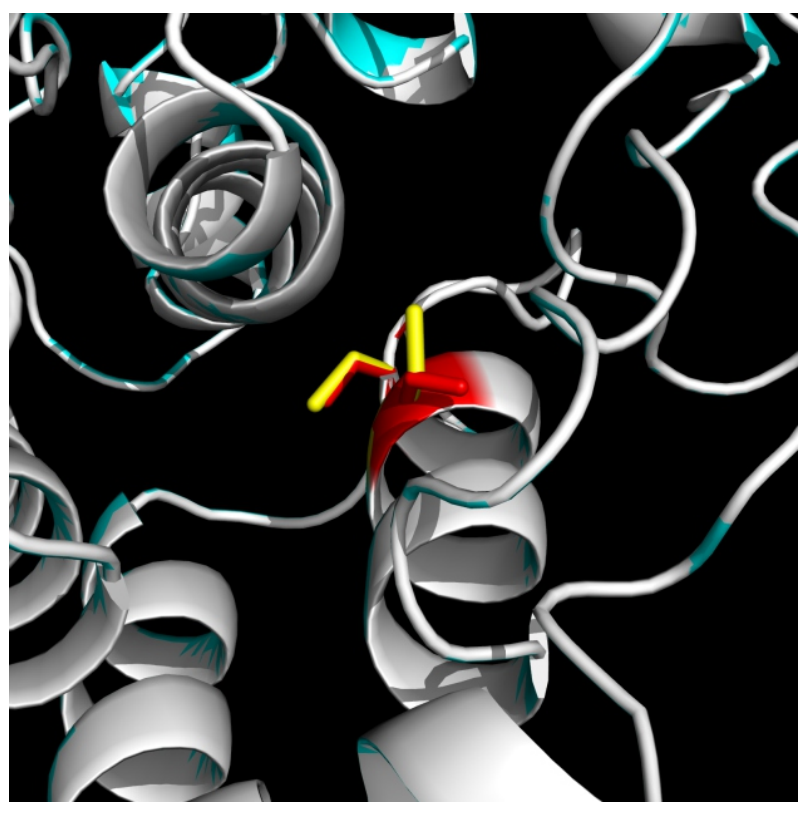

Supplemental figure 4. Mutation of kinase domain S-acylation site cysteines to serine in FLS2 is not predicted to affect kinase domain structure. A. Superimposition of the modelled structures of FLS2 (white) and FLS2 $\mathrm{C}^{1132,1135} \mathrm{~S}$ (blue) kinase domains. B. Zoomed in view of Cys1132,1135 in FLS2 (yellow) and substituted serine (red) residues in FLS2 ${ }^{1132,1135}$ S. Only the proton of Ser1132 is predicted to diverge from the FLS2 structure, being rotated by $\sim 110$ degrees compared to the original cysteine. This rotation does not affect the position or packing of any other amino acid. 


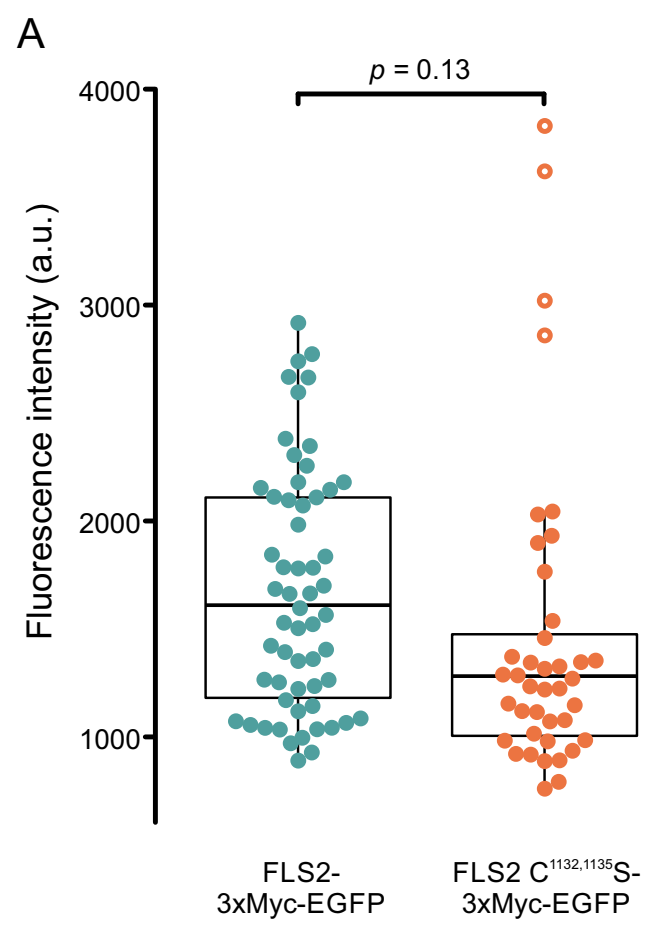

$\mathrm{B}$

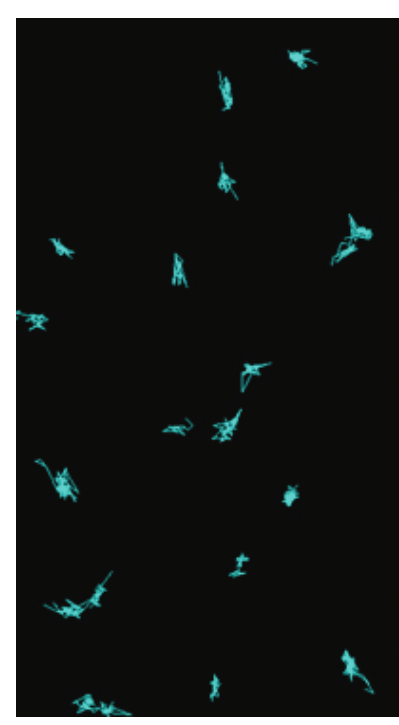

FLS2-

3xMyc-EGFP

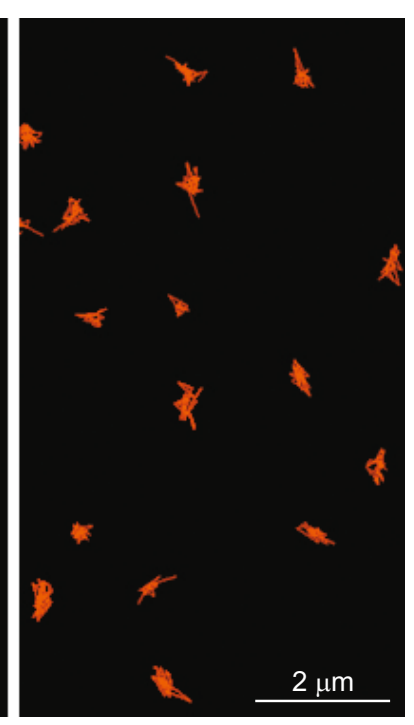

FLS2 $C^{1132,1135} S-$ 3xMyc-EGFP
C

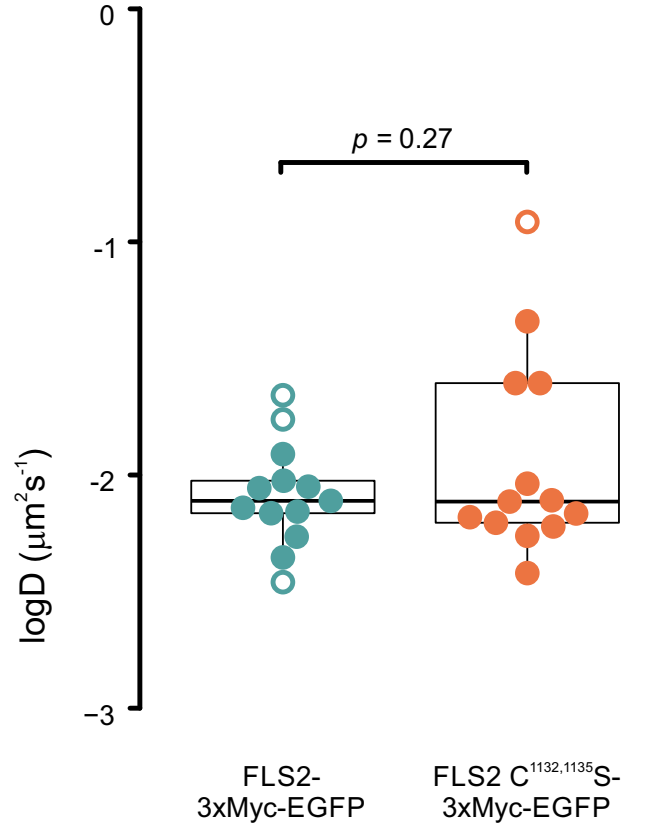

Supplemental figure 5. FLS2-3xMyc-GFP and FLS2 $C^{1132,1135}$ S-3xMyc-GFP behave similarly when expressed in $N$. benthamiana in the absence of flg22. A. Fluorcence intensity measurements at the plasma membrane of single cells using TIRF microscopy. Box plot shows median and IQR, whiskers indicate $1.5 \times$ IQR. FLS2-3xMyc-GFP $\mathrm{n}=59$, FLS2 $\mathrm{C}^{1132,1135} \mathrm{~S}-3 \mathrm{xMyc}-\mathrm{GFP} \mathrm{n}=42$. $p$ value calculated using Student's t-test. Statistical outliers are indicated by open circles. B. Single particle tracking of FLS2-3xMyc-GFP and FLS2 $\mathrm{C}^{1132,1135} \mathrm{~S}-3 \mathrm{xMyc}-\mathrm{GFP}$ at the plasma membrane using TIRF microscopy. C. Quantification of data in B. Box plot shows median and IQR, whiskers indicate $1.5 \times I Q R$. $n=13, p$ value calculated using Student's t-test. Statistical outliers are indicated by open circles. 
bioRxiv preprint doi: https://doi.org/10.1101/2021.08.30.457756; this version posted August 31, 2021. The copyright holder for this preprint (which was not certified by peer review) is the author/funder, who has granted bioRxiv a license to display the preprint in perpetuity. It is A made available under aCC-BY-NC-ND 4.0 International license.
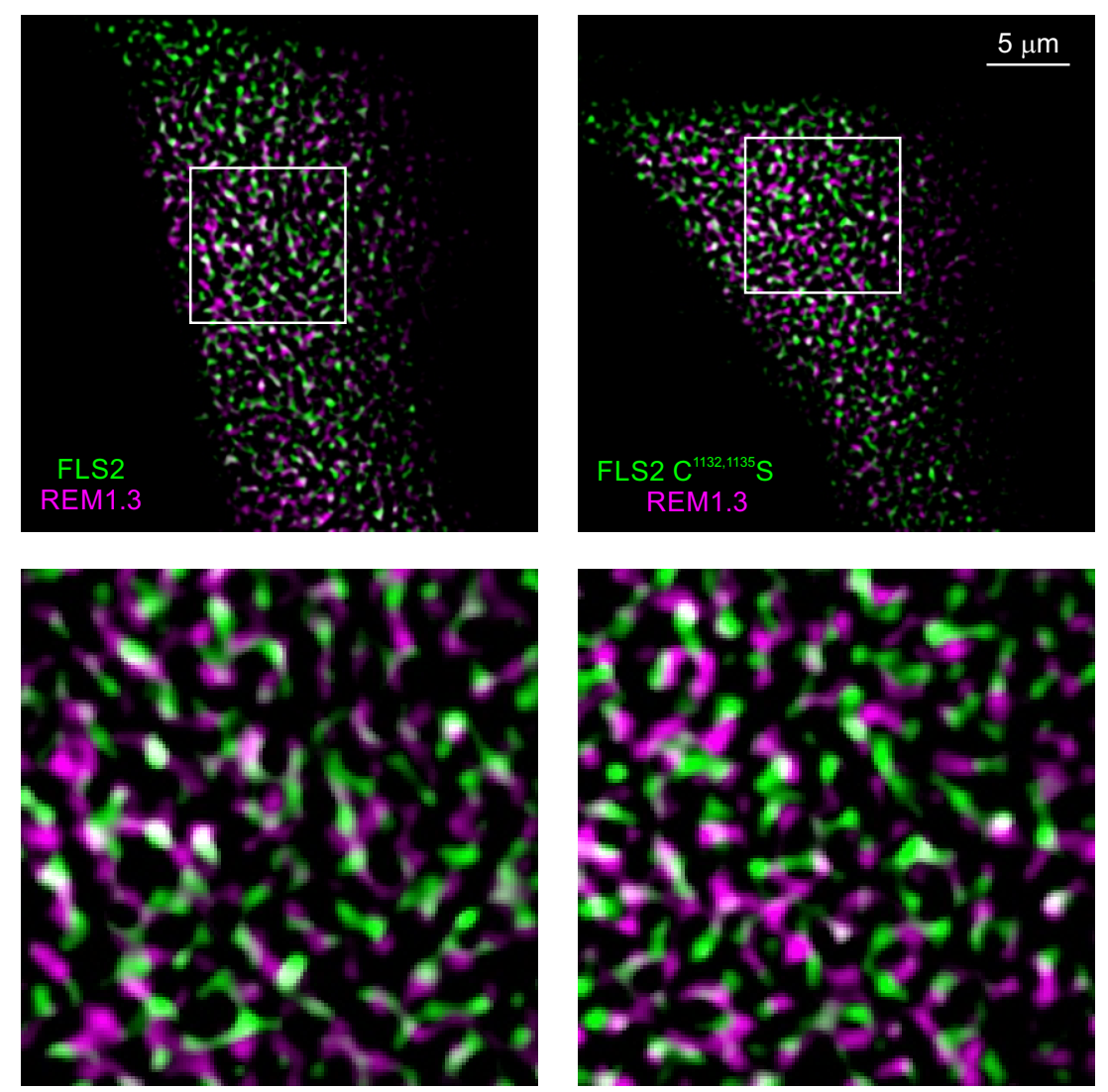

B

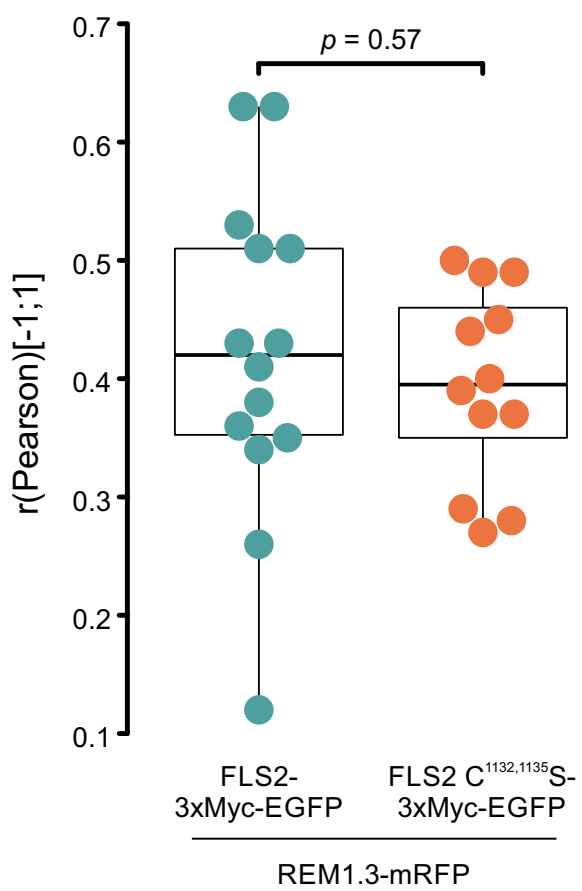

C

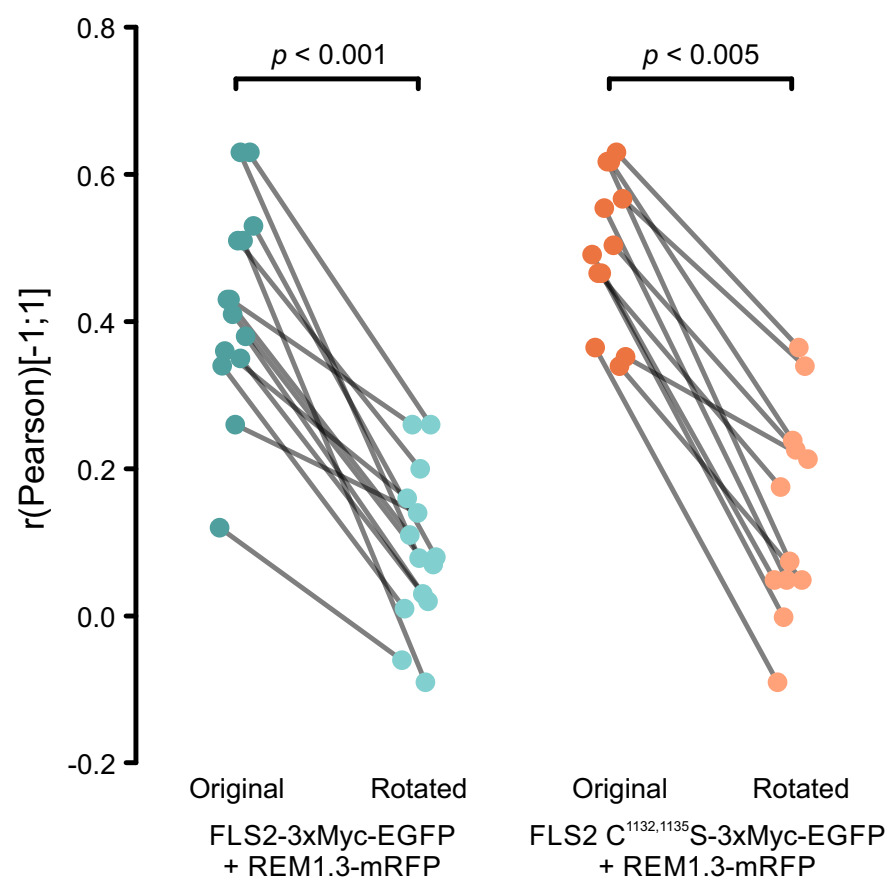

Supplemental figure 6. FLS2-3xMyc-GFP and FLS2 $C^{1132,1135}$ S-3xMyc-GFP form nanodomains in the plasma membrane and show similar co-localisation with mRFP-REM1.3 nanodomains when transiently expressed in $N$. benthamiana in the absence of flg22. A. Representative micrographs of FLS2-3xMyc-GFP and FLS2 $\mathrm{C}^{1132,1135}$ S-3xMyc-GFP (green) co-localisation with mRFP-REM1.3 (magenta) at the plasma membrane of single epidermal cells using TIRF microscopy. B. Quantification of FLS2-3xMyc-GFP or FLS2 C1132,1135S-3xMyc-GFP co-localisation with mRFPREM1.3 at the plasma membrane of single epidermal cells. FLS2-3xMyc-GFP $n=14$, FLS2 $\mathrm{C}^{1132,1135} \mathrm{~S}-3 \mathrm{xMyc}-\mathrm{GFP} \mathrm{n}=12$. Box plot shows median and IQR, whiskers indicate $1.5 \times I Q R$. $p$ value calculated using Student's t-test. C. To determine whether measured co-localisation values shown in $\mathrm{B}$ (original) were significant, co-localisation analysis was repeated after rotation of the mRFPREM1.3 image by 90 degrees (rotated). In all cases, co-localisation was reduced and overall significantly different, indicating that the co-localisation observed in B is both specific and significant. $p$ values were calculated using Student's t-test. 


\section{Materials and Methods \\ Eliciting peptides}

Flg22 peptide (QRLSTGSRINSAKDDAAGLQIA) was synthesised by Dundee Cell Products (Dundee, UK). Elf18 peptide (Ac-SKEKFERTKPHVNVGTIG) was synthesised by Peptide Protein Research Ltd. (Bishops Waltham, UK).

\section{Cloning and constructs}

Fully functional $F L S 2_{\text {pro: }} F L S 2$ was made using the described FLS2 promoter and open reading frame (18) with stop codon cloned into pENTR D-TOPO (19). All FLS2 mutant variants used were based on this construct and were generated using Q5 site directed mutagenesis kit (NEB) according to the manufacturer's guidelines. FLS2 $2_{\text {pro }}$ FLS2-3xMYC-EGFP and FLS2 ${ }_{\text {pro: }}$ FLS2 $C^{1132,1135} S-3 \times M Y C-E G F P$ were made by yeast recombinatorial cloning using a 3xMYC-EGFP PCR fragment amplified from $F L S 2_{\text {pro }}$ :FLS2$3 \times M Y C-E G F P(20)$ recombined with pENTR D-TOPO FLS2 pro:FLS2 or pENTR D-TOPO FLS2 pro:FLS2 $\mathrm{C}^{1132,1135} \mathrm{~S}$. Entry clones were recombined into pK7WG,0 (21) using Gateway technology (ThermoFisher) to generate expression constructs. Expression constructs were transformed into Agrobacterium tumefaciens strain GV3101 pMP90 (22) for transformation of either Arabidopsis or Nicotiana benthamiana.

\section{Plant lines and growth conditions}

All Arabidopsis lines were in the Col-0 accession background. The fls2 (18), bak1-4 (23) and chc2-1 (24) mutants have all been described previously. Transgenic fls2/FLS2 pro:FLS2 (19) and fls2/FLS2 pro:FLS2 $C^{1132,1135} S$ mutant variant lines were generated by Agrobacterium-mediated floral dip transformation (25). $T_{3}$ homozygous plants were used for all experiments. Plant material for experiments was grown on $0.5 x$ MS medium, $0.8 \%$ phytagar under $16: 8$ light:dark cycles at $20^{\circ} \mathrm{C}$ in MLR-350 growth chambers (Panasonic). For transient expression Nicotiana benthamiana plants were grown in 16:8 light:dark cycles at $24{ }^{\circ} \mathrm{C}$ and used at 4-5 weeks old. A. tumefaciens mediated transient expression was performed as described (26) using an OD600 of 0.1 of each expression construct alongside the p19 silencing suppressor at an OD600 of 0.1. Tissue was harvested $48-60$ hours post infiltration.

\section{Seedling growth inhibition}

For each biological replicate four days post-germination, 10 seedlings of the named genotypes were transferred to 12-well plates (5 seedlings per well), ensuring the cotyledons were not submerged. Wells contained $2 \mathrm{~mL}$ of $0.5 x \mathrm{MS}$ liquid medium with or without $1 \mu \mathrm{M}$ flg22. Seedlings were incubated for 10 days and the fresh weight of pooled seedlings in each genotype for each treatment measured and an average taken. Flg22- treated/untreated weights for each genotype were calculated and presented data is an average of these data over three biological repeats. Fully independent biological repeats were performed over a period of 6 months with each genotype only being present once in each repeat.

\section{MAPK activation}

Essentially as for (27); 6 seedlings of each genotype 10 days post germination were treated with 100 $\mathrm{nM}$ flg22 for the indicated times in $2 \mathrm{~mL} 0.5 \times \mathrm{MS}$ medium. The 6 seedlings from each genotype at each time point for each treatment were pooled before further analysis. Fully independent biological repeats were performed over a period of 2 years with each genotype only being present once in each repeat. 


\section{Reactive oxygen species production}

Protocol based on Mersmann et al. (2010). Essentially, 10 seedlings of each genotype were grown for 14 days in $100 \mu \mathrm{L}$ of $0.5 \mathrm{x}$ MS medium with $0.5 \%$ sucrose, in 96-well plates (PerkinElmer). Conditions were maintained at $22^{\circ} \mathrm{C}$ with 12:12 light:dark cycles. Growth medium was exchanged for water with $10 \mathrm{nM}$ flg22 for 1 hour, before replacing with water for a further 1 hour. ROS burst was then induced by replacing with a solution containing $100 \mathrm{nM}$ flg22, $400 \mathrm{nM}$ luminol (Fluka), and $20 \mu \mathrm{g} / \mathrm{mL}$ peroxidase (Sigma). Luminescence in each well was measured every 2 minutes in a Varioskan Lux (Thermo Fisher) for 30 cycles (approx. 1 hour total).

\section{S-acylation assays}

S-acylation assays using acyl-biotin exchange (ABE) were performed exactly as described (28). For flg22-dependent changes in FLS2 S-acylation, 7 seedlings 10 days post germination were transferred to each well of 12-well plates. Each well contained $2 \mathrm{~mL} 0.5 \times$ MS liquid medium. Seedling were incubated for 24 hours on an orbital mixer (Luckham R100/TW Rotatest Shaker, $38 \mathrm{~mm}$ orbit at 75 RPM). Thereafter, $100 \mu \mathrm{L}$ of $0.5 \times$ MS media containing flg22 was added to give a final flg22 concentration of $10 \mu \mathrm{M}$. Seedlings were incubated with continued mixing for the indicated times before harvesting.

\section{Co-immunoprecipitation assays using IGEPAL CA-630}

Seedlings grown on solid $1 / 2 \mathrm{MS}$ for 30-35 days were transferred to wells of a 6 -well plates and grown for 7 days in $1 / 2 \mathrm{MS} 2 \mathrm{mM}$ MES-KOH, pH 5.8. Thereafter, the seedlings were transferred in beakers containing $40 \mathrm{~mL}$ of $1 / 2 \mathrm{MS} 2 \mathrm{mM}$ MES-KOH, pH 5.8 and subsequently treated with sterile mQ water with or without flg22 (final concentration of $100 \mathrm{nM}$ ) and incubated for 10 minutes. The seedlings

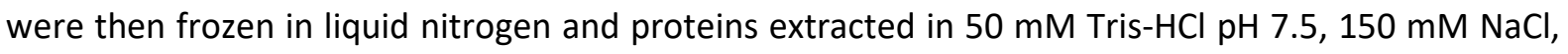
$10 \%$ glycerol, $5 \mathrm{mM}$ dithiothreitol, 1\% protease inhibitor cocktail (Sigma Aldrich), $2 \mathrm{mM} \mathrm{Na}_{2} \mathrm{MoO}_{4}, 2.5$ $\mathrm{mM} \mathrm{NaF}, 1.5 \mathrm{mM}$ activated $\mathrm{Na}_{3} \mathrm{VO}_{4}, 1 \mathrm{mM}$ phenylmethanesulfonyl fluoride and 0.5\% IGEPAL for 40 minutes at $4{ }^{\circ} \mathrm{C}$. Lysates were clarified at $10,000 \mathrm{~g}$ for 20 minutes at $4{ }^{\circ} \mathrm{C}$ and the supernatants were filtered through miracloth. For immunoprecipitations, $\alpha$-rabbit Trueblot agarose beads (eBioscience) coupled with $\alpha$-FLS2 antibodies (29) were incubated with the crude extract for 3 hours at $4{ }^{\circ} \mathrm{C}$. Subsequently, beads were washed 3 times $(50 \mathrm{mM}$ Tris- $\mathrm{HCl} \mathrm{pH} 7.5,150 \mathrm{mM} \mathrm{NaCl}, 1 \mathrm{mM}$ phenylmethanesulfonyl fluoride, $0.1 \%$ IGEPAL) before adding Laemmli buffer and incubating for 10 minutes at $95^{\circ} \mathrm{C}$. Protein samples were separated in $10 \%$ bisacrylamide gels at $150 \mathrm{~V}$ for approximately 2 hours and transferred into activated PVDF membranes at $100 \mathrm{~V}$ for 90 minutes. Immunoblotting was performed with antibodies diluted in blocking solution ( $5 \%$ fat-free milk in TBS with $0.1 \%$ (v/v) Tween20). Antibodies used in this study: $\alpha$-BAK1 (30) (1:5000); $\alpha$-FLS2 (29) (1:1000); $\alpha$-BAK1 pS612 (31) (1:3000). Blots were developed with Pierce ECL/ ECL Femto Western Blotting Substrate (Thermo Scientific). The following secondary antibodies were used: anti-rabbit IgG-HRP Trueblot (Rockland, 188816-31, dilution 1:10000) for detection of FLS2-BAK1 co-immunoprecipitation or anti-rabbit IgG (whole molecule)-HRP (A0545, Sigma, dilution 1:10000) for all other western blots.

\section{Co-immunoprecipitation assays using Diisobutylene-maleic acid (DIBMA)}

For each genotype, $2 \times 10$ seedlings 10 days post-germination were transferred to each well of 12-well plate containing $2 \mathrm{~mL} 0.5 \times \mathrm{MS}$ liquid medium and incubated for 24 hours on an orbital mixer (Luckham R100/TW Rotatest Shaker, $38 \mathrm{~mm}$ orbit at 75 RPM). Thereafter, $100 \mu \mathrm{L}$ of $0.5 \times$ MS media containing 
flg22 was added to give a final flg22 concentration of $10 \mu \mathrm{M}$. The seedlings were further incubated with continued mixing for 20 minutes prior to harvesting and blotting dry. Tissue was lysed in $500 \mu \mathrm{L}$ of lysis buffer (50 mM Tris-HCl pH 7.2, 10\% v/v glycerol, $150 \mathrm{mM} \mathrm{NaCl}, 1 \% \mathrm{w} / \mathrm{v}$ DIBMA (Anatrace BMA101), with protease inhibitors (1\% v/v, Sigma P9599)) and incubated at room temperature for 1 hour with gentle end-over-end mixing. The lysate was centrifuged at 5,000 g for 1 minute and the supernatant filtered through 2 layers of miracloth and combined with an additional $500 \mu \mathrm{L}$ of filtered lysis buffer (without DIMBA). The clarified lysate was further centrifuged at $16,000 \mathrm{~g}$ for 1 minute and the supernatant applied to Amicon $0.5 \mathrm{~mL} 100 \mathrm{kDa}$ MWCO spin filtration columns an centrifuged at $14,000 \mathrm{~g}$ until the retentate was $<50 \mu \mathrm{L}$. The retentate was diluted to $500 \mu \mathrm{L}$ with IP buffer $(50 \mathrm{mM}$ Tris- $\mathrm{HCl} \mathrm{pH} \mathrm{7.2,} \mathrm{10 \%} \mathrm{glycerol,} 200 \mathrm{mM} \mathrm{L-arginine,} \mathrm{with} \mathrm{protease} \mathrm{inhibitor} \mathrm{(0.5 \%} \mathrm{v/v,} \mathrm{Sigma} \mathrm{P9599)} \mathrm{and}$ centrifuged at $14,000 \mathrm{~g}$ until the retentate was $<50 \mu \mathrm{L}$. The spin column was inverted and eluted into a $1.5 \mathrm{~mL}$ microfuge tube by centrifugation at $100 \mathrm{~g}$ for 1 minute The eluate was diluted to $500 \mu \mathrm{L}$ with IP buffer, of which $20 \mu \mathrm{L}$ was retained as an input control. Magnetic protein A beads $(20 \mu \mathrm{L}$ per IP reaction) were coated with $5 \mu \mathrm{g} \alpha \mathrm{FLS} 2$ antibody overnight at $4{ }^{\circ} \mathrm{C}$. The resulting beads were washed for 5 minutes with IP buffer containing $0.5 \mathrm{M} \mathrm{NaCl}$ followed by 2 washes with IP buffer, and resuspended in IP buffer to $100 \mu \mathrm{L}$ per IP reaction. The resulting FLS2-coated magnetic protein $\mathrm{A}$ beads were added to the DIBMA solubilised protein solution and incubated for 3 hours at room temperature with end-over-end mixing. Thereafter, the beads were washed thrice with IP buffer and resuspended in $30 \mu \mathrm{L} 2 \mathrm{x}$ LDS sample buffer with 2-mercaptoethanol, and incubated at $65{ }^{\circ} \mathrm{C}$ for 5 minutes with shaking at 1000 RPM. The samples were separated on a 7.5\% SDS-PAGE gel prior to transfer to PVDF and western blotting.

\section{Detergent resistant membrane preparation}

To evaluate flg22-dependent changes in FLS2 detergent resistant membrane occupancy, 7 seedlings 10 days post-germination were transferred to each well of a 12-well plate, of which each well contained $2 \mathrm{~mL} 0.5 \times$ MS liquid medium. Seedlings were incubated for 24 hours on an orbital mixer (Luckham R100/TW Rotatest Shaker, $38 \mathrm{~mm}$ orbit at 75 RPM), after which $100 \mu \mathrm{L}$ of $0.5 \times$ MS media containing flg22 was added to give a final flg22 concentration of $10 \mu \mathrm{M}$. The seedlings were further incubated with continuous mixing as before for 20 minutes before harvesting and snap freezing in liquid nitrogen. All subsequent steps were performed at $4{ }^{\circ} \mathrm{C}$ or on ice. The seedlings were then lysed in $0.5 \mathrm{~mL}$ ice cold 1\% (v/v) IGEPAL CA-630 in $25 \mathrm{mM}$ Tris-HCl pH 7.4, $150 \mathrm{mM} \mathrm{NaCl}, 2 \mathrm{mM}$ EDTA, and $0.1 \%(\mathrm{v} / \mathrm{v})$ protease inhibitors (Sigma-Aldrich, P9599). Lysates were clarified at $500 \mathrm{~g}$ and filtered through 1 layer of miracloth. The filtrate was centrifuged at $16,000 \mathrm{~g}$ for 30 minutes and thesupernatant retained as a detergent soluble fraction (DSM) and mixed 3:1 with 4x reducing (2mercaptoethanol) LDS sample buffer. The detergent resistant pellet (DRM) was gently washed with 1 $\mathrm{mL}$ lysis buffer, centrifuged at 16,000 $\mathrm{g}$ for 5 minutes, and the supernatant discarded. The resulting pellet was resuspended in $27 \mu \mathrm{L}$ of 3:1 lysis buffer: $4 x$ reducing LDS sample buffer, after which $25 \mu \mathrm{L}$ of the DRM and DSM were separated by 7.5\% SDS-PAGE and probed using anti-FLS2 polyclonal antibody as described (28).

\section{Variable Angle - Total Internal Reflection Fluorescence (VA-TIRF) microscopy}

VA-TIRF microscopy was performed using an inverted Leica GSD equipped with a 160x objective (NA $=1.43$, oil immersion), and an Andor iXon Ultra 897 EMCCD camera. Images were acquired by illuminating samples with a $488 \mathrm{~nm}$ solid state diode laser, a cube filter with an excitation filter 488/10 and an emission filter 535/50 for FLS2-GFP, and a $532 \mathrm{~nm}$ solid state diode laser, a cube filter with an 
excitation filter 532/10 and an emission filter 600/100 for mRFP-REM1.3. Optimum critical angle was determined as giving the best signal-to-noise.

\section{Single particle tracking analysis}

Nicotiana benthamiana plants (14-21 days old) were infiltrated with Agrobacterium tumefaciens (strain GV3101) solution of OD $=0.15$ and imaged 32 to 40 hours post infiltration. For single particle tracking experiments, image time series were recorded at 20 frames per second (50 ms exposure time) by VA-TIRFM. Analyses were carried out as previously described (32), using the plugin TrackMate 2.7.4 in Fiji. Single particles were segmented frame-by-frame by applying a Laplacian of Gaussian filter and estimated particle size of $0.4 \mu \mathrm{m}$. Individual single particle were localized with sub-pixel resolution using a built-in quadratic fitting scheme. Single particle trajectories were reconstructed using a simple linear assignment problem (33) with a maximal linking distance of $0.4 \mu \mathrm{m}$ and without gap-closing. Only tracks with at least ten successive points (tracked for $500 \mathrm{~ms}$ ) were selected for further analysis. Diffusion coefficients of individual particles were determined using TraJClassifier (34). For each particle, the slope of the first four time points of their mean square displacement (MSD) plot was used to calculate their diffusion coefficient according to the following equation: MSD $=(x-x 0) 2+(y-y 0) 2$ and $\mathrm{D}=\mathrm{MSD} / 4 \mathrm{t}$, where $\mathrm{x} 0$ and $\mathrm{y} 0$ are the initial coordinates, $\mathrm{x}$ and $\mathrm{y}$ are the coordinates at any given time, and $t$ is the time frame.

\section{Co-localization analyses}

Nicotiana benthamiana plants (14-21 days old) were infiltrated with Agrobacterium tumefaciens (strain GV3101) solution of OD $=0.2$ and imaged 48 hours post infiltration. Images were recorded by VA-TIRFM using $250 \mathrm{~ms}$ exposure time. As previously reported (35), we emphasised cluster formation in the presented images by using the 'LoG3D' plugin (36). Quantitative co-localization analyses of the FLS2-GFP and mRFP-REM1.3 were carried out as previously described (35), with minor modification. Using FiJi, images were subjected to a background subtraction using the "Rolling ball" method (radius $=20$ pixels) and smoothed. We selected regions of TIRF micrographs with homogeneous illumination for both FLS2-GFP and mRFP-REM1.3. The Pearson co-localization coefficients were assessed using the JACoP plugin of FIJI (37). For comparison, we determined values of correlation, which could be observed by chance by calculating the Pearson coefficient after flipping one of the two images.

\section{Western blotting}

FLS2 was detected using rabbit polyclonal antisera raised against the C-terminus of FLS2 as previously described $(28,38)$. Anti-p44/42 MAPK (Erk1/2) (Cell Signalling Technology \#9102) was used to detect phosphorylated MAPK3/6 according to manufacturer's recommendations at 1:2000 dilution. Rabbit polyclonal antibodies against BAK1 were as described (30) or obtained from Agrisera (AS12 1858) and used at 1:5000 dilution. BAK1 phospho-S612 was detected using polyclonal rabbit antisera as described (31). HRP conjugated secondary antibodies were used to visualise antibody reacting proteins, and Clean-Blot HRP (Thermo Fisher) secondary antibody was used for immunoprecipitation experiments. Western blots were developed using SuperSignal West pico and femto in a 3:1 ratio by volume and signal captured using a Syngene G:box storm imager and quantitative photon count data stored as Syngene SGD files. Signal intensity was quantified from SGD files using Syngene GeneTools software.

\section{Gene expression analysis}


Ten seedlings of each genotype 10 days post-germination were treated with $1 \mu \mathrm{M}$ flg22 or water for the indicated times. The 10 seedlings from each genotype/treatment at each time point for each treatment were pooled before further analysis. RNA was extracted using RNAeasy Plant kit with on column DNAse digestion according to the manufacturer's instructions (Qiagen). Two micrograms RNA was reverse transcribed using a High-Capacity cDNA Reverse Transcription kit (Applied Biosystems). All transcripts were amplified using validated gene-specific primers (19). Expression levels were normalized against PEX4 (At5g25760) (39). Each sample was analyses in triplicate (technical repeats) for each primer pair within each biological repeat. Relative quantification (RQ) was achieved using the $\Delta \Delta_{\text {ст }}$ (comparative cycle threshold) method (40). Significant differences between samples were determined from a $95 \%$ confidence interval calculated using the t-distribution. Fully independent biological repeats were performed over a period of 2 years with each genotype only being present once in each repeat.

\section{Bacterial infection assays}

Infection assays of Arabidopsis lines by Pseudomonas syringae pv. tomato DC3000 were performed using seedling flood inoculation assays as described (41).

\section{Structural modelling of FLS2 kinase domain}

The FLS2 intracellular domain (amino acids 831-1173) was submitted to the phyre2 (42) server (http://www.sbg.bio.ic.ac.uk/phyre2/) in default settings. The solved BIR2 kinase domain structure (PDB 4L68, residues 272-600) (43) was identified as the best match and FLS2 residues 841-1171 were successfully modelled onto the BIR2 structure (confidence $100 \%$, coverage $89 \%$ ). Cys to Ser mutational effects were modelled using Missense3D (44) in default settings. 


\section{References and notes}

1. L. Gomez-Gomez, T. Boller, FLS2: an LRR receptor-like kinase involved in the perception of the bacterial elicitor flagellin in Arabidopsis. Mol Cell 5, 1003-1011 (2000).

2. C. H. Hurst et al., Juxta-membrane S-acylation of plant receptor-like kinases is likely fortuitous and does not necessarily impact upon function. Sci Rep 9, 12818 (2019).

3. P. A. Hemsley, T. Weimar, K. S. Lilley, P. Dupree, C. S. Grierson, A proteomic approach identifies many novel palmitoylated proteins in Arabidopsis. New Phytologist 197, 805-814 (2013).

4. C. H. Hurst, P. A. Hemsley, Current perspective on protein S-acylation in plants: more than just a fatty anchor? Journal of Experimental Botany 66, 1599-1606 (2015).

5. D. Turnbull, P. A. Hemsley, Fats and function: protein lipid modifications in plant cell signalling. Curr Opin Plant Biol 40, 63-70 (2017).

6. C. Zipfel et al., Perception of the bacterial PAMP EF-Tu by the receptor EFR restricts Agrobacterium-mediated transformation. Cell 125, 749-760 (2006).

7. Materials and methods are available as supplementary materials at the Science website.

8. M. Mbengue et al., Clathrin-dependent endocytosis is required for immunity mediated by pattern recognition receptor kinases. Proc Natl Acad Sci U S A 113, 11034-11039 (2016).

9. M. Kumar, P. Carr, S. Turner, An atlas of Arabidopsis protein S-Acylation reveals its widespread role in plant cell organisation of and function. bioRxiv, (2020).

10. A. O. Oluwole et al., Solubilization of Membrane Proteins into Functional Lipid-Bilayer Nanodiscs Using a Diisobutylene/Maleic Acid Copolymer. Angew Chem Int Ed Eng/ 56, 19191924 (2017).

11. A. Perraki et al., Phosphocode-dependent functional dichotomy of a common co-receptor in plant signalling. Nature 561, 248-252 (2018).

12. J. Wang et al., Allosteric receptor activation by the plant peptide hormone phytosulfokine. Nature 525, 265-268 (2015).

13. N. F. Keinath et al., PAMP-induced changes in plasma membrane compartmentalization reveal novel components of plant immunity. J Biol Chem 285, 39140-39149 (2010).

14. Y. Cui et al., Sterols regulate endocytic pathways during flg22-induced defense responses in Arabidopsis. Development 145, (2018).

15. M. Stegmann et al., The receptor kinase FER is a RALF-regulated scaffold controlling plant immune signaling. Science 355, 287-289 (2017).

16. L. Abrami, S. H. Leppla, F. G. van der Goot, Receptor palmitoylation and ubiquitination regulate anthrax toxin endocytosis. J Cell Biol 172, 309-320 (2006).

17. D. Chen et al., S-acylation of P2K1 mediates extracellular ATP-induced immune signaling in Arabidopsis. Nat Commun 12, 2750 (2021).

18. C. Zipfel et al., Bacterial disease resistance in Arabidopsis through flagellin perception. Nature 428, 764-767 (2004).

19. C. H. Hurst et al., Variable Effects of C-Terminal Fusions on FLS2 Function: Not All Epitope Tags Are Created Equal. Plant Physiol 177, 522-531 (2018).

20. S. Robatzek, D. Chinchilla, T. Boller, Ligand-induced endocytosis of the pattern recognition receptor FLS2 in Arabidopsis. Genes Dev 20, 537-542 (2006).

21. M. Karimi, D. Inze, A. Depicker, GATEWAY vectors for Agrobacterium-mediated plant transformation. Trends Plant Sci 7, 193-195 (2002). 
22. C. Koncz, J. Schell, The promoter of TL-DNA gene 5 controls the tissue-specific expression of chimaeric genes carried by a novel type of Agrobacterium binary vector. Mol. Gen. Genet. 204, (1986).

23. B. Kemmerling et al., The BRI1-associated kinase 1, BAK1, has a brassinolide-independent role in plant cell-death control. Curr Biol 17, 1116-1122 (2007).

24. M. Mbengue et al., Clathrin-dependent endocytosis is required for immunity mediated by pattern recognition receptor kinases. Proc Natl Acad Sci U S A 113, 11034-11039 (2016).

25. S. J. Clough, A. F. Bent, Floral dip: a simplified method for Agrobacterium-mediated transformation of Arabidopsis thaliana. Plant J 16, 735-743 (1998).

26. D. Turnbull et al., RXLR Effector AVR2 Up-Regulates a Brassinosteroid-Responsive bHLH Transcription Factor to Suppress Immunity. Plant Physiol 174, 356-369 (2017).

27. B. Schwessinger et al., Phosphorylation-dependent differential regulation of plant growth, cell death, and innate immunity by the regulatory receptor-like kinase BAK1. PLoS Genet 7, e1002046 (2011).

28. C. H. Hurst, D. Turnbull, F. Plain, W. Fuller, P. A. Hemsley, Maleimide scavenging enhances determination of protein S-palmitoylation state in acyl-exchange methods. Biotechniques 62 , 69-75 (2017).

29. D. Chinchilla et al., A flagellin-induced complex of the receptor FLS2 and BAK1 initiates plant defence. Nature 448, 497-500 (2007).

30. M. Roux et al., The Arabidopsis leucine-rich repeat receptor-like kinases BAK1/SERK3 and BKK1/SERK4 are required for innate immunity to hemibiotrophic and biotrophic pathogens. Plant Cell 23, 2440-2455 (2011).

31. A. Perraki et al., Phosphocode-dependent functional dichotomy of a common co-receptor in plant signalling. Nature 561, 248-252 (2018).

32. J. Gronnier et al., FERONIA regulates FLS2 plasma membrane nanoscale dynamics to modulate plant immune signaling. bioRxiv, (2020).

33. K. Jaqaman et al., Robust single-particle tracking in live-cell time-lapse sequences. Nat Methods 5, 695-702 (2008).

34. T. Wagner, A. Kroll, C. R. Haramagatti, H. G. Lipinski, M. Wiemann, Classification and Segmentation of Nanoparticle Diffusion Trajectories in Cellular Micro Environments. PLoS One 12, e0170165 (2017).

35. C. A. Bucherl et al., Plant immune and growth receptors share common signalling components but localise to distinct plasma membrane nanodomains. Elife 6, e25114 (2017).

36. D. Sage, F. R. Neumann, F. Hediger, S. M. Gasser, M. Unser, Automatic tracking of individual fluorescence particles: application to the study of chromosome dynamics. IEEE Transactions on Image Processing 14, 1372-1383 (2005).

37. S. Bolte, F. P. Cordelieres, A guided tour into subcellular colocalization analysis in light microscopy. J Microsc 224, 213-232 (2006).

38. A. Heese et al., The receptor-like kinase SERK3/BAK1 is a central regulator of innate immunity in plants. Proc Natl Acad Sci U S A 104, 12217-12222 (2007).

39. D. L. Wathugala et al., The Mediator subunit SFR6/MED16 controls defence gene expression mediated by salicylic acid and jasmonate responsive pathways. New Phytologist 195, 217-230 (2012).

40. T. D. Schmittgen, K. J. Livak, Analyzing real-time PCR data by the comparative $C(T)$ method. Nat Protoc 3, 1101-1108 (2008). 
41. Y. Ishiga, T. Ishiga, S. R. Uppalapati, K. S. Mysore, Arabidopsis seedling flood-inoculation technique: a rapid and reliable assay for studying plant-bacterial interactions. Plant Methods 7, 32 (2011).

42. L. A. Kelley, S. Mezulis, C. M. Yates, M. N. Wass, M. J. Sternberg, The Phyre2 web portal for protein modeling, prediction and analysis. Nat Protoc 10, 845-858 (2015).

43. B. S. Blaum et al., Structure of the pseudokinase domain of BIR2, a regulator of BAK1-mediated immune signaling in Arabidopsis. J Struct Biol 186, 112-121 (2014).

44. S. Ittisoponpisan et al., Can Predicted Protein 3D Structures Provide Reliable Insights into whether Missense Variants Are Disease Associated? J Mol Biol 431, 2197-2212 (2019). 